\title{
Differential Spatial Resection - Pose Estimation Using a Single Local Image Feature
}

\author{
Kevin Köser and Reinhard Koch \\ Institute of Computer Science \\ Christian-Albrechts-University of Kiel \\ 24098 Kiel, Germany \\ \{koeser,rk\}@mip.informatik . uni-kiel.de
}

\begin{abstract}
Robust local image features have been used successfully in robot localization and camera pose estimation; region tracking using affine warps is considered state of the art also for many years. Although such correspondences provide a warp of the local image region and are quite powerful, in direct pose estimation they are so far only considered as points and therefore three of them are required to construct a camera pose. In this contribution we show how it is possible to directly compute a pose based upon one such feature, given the plane in space where it lies. This differential correspondence concept exploits the texture warp and has recently gained attention in estimation of conjugate rotations. The approach can also be considered as the limiting case of the well-known spatial resection problem when the three $3 \mathrm{D}$ points approach each other infinitesimally close. We show that the differential correspondence is more powerful than conic correspondences while its exploitation requires nothing more complicated than the roots of a third order polynomial. We give a detailed sensitivity analysis, a comparison against state-of-the-art pose estimators and demonstrate real-world applicability of the algorithm based on automatic region recognition.
\end{abstract}

\section{Introduction}

Since the first description of spatial resection from 3 points by Grunert [7] in 1841, many people have worked on pose estimation or the so called P3P problem [5]316 6 . PnP stands for pose estimation from $n$ points and is underconstrained for $n<3$ unless further information is incorporated. In this work we derive how a variation of the problem may be solved, namely when only a single affine image feature (cf. to 24 for a discussion) can be identified with a known 3D space surface with orthophoto texture. Additionally to the traditionally used 2D-3D point correspondence, such an image-model relation provides a local linear texture warp between the image and an orthophoto of the surface. This warp can be interpreted as the Jacobian of the perspectivity between the image and the 3D surface's tangent plane and we show that it determines the open degrees of freedom. The novel approach allows to estimate a perspective camera's pose based upon only one image-model correspondence, which is particularly interesting in

D. Forsyth, P. Torr, and A. Zisserman (Eds.): ECCV 2008, Part IV, LNCS 5305, pp. 312 325, 2008.

(C) Springer-Verlag Berlin Heidelberg 2008 

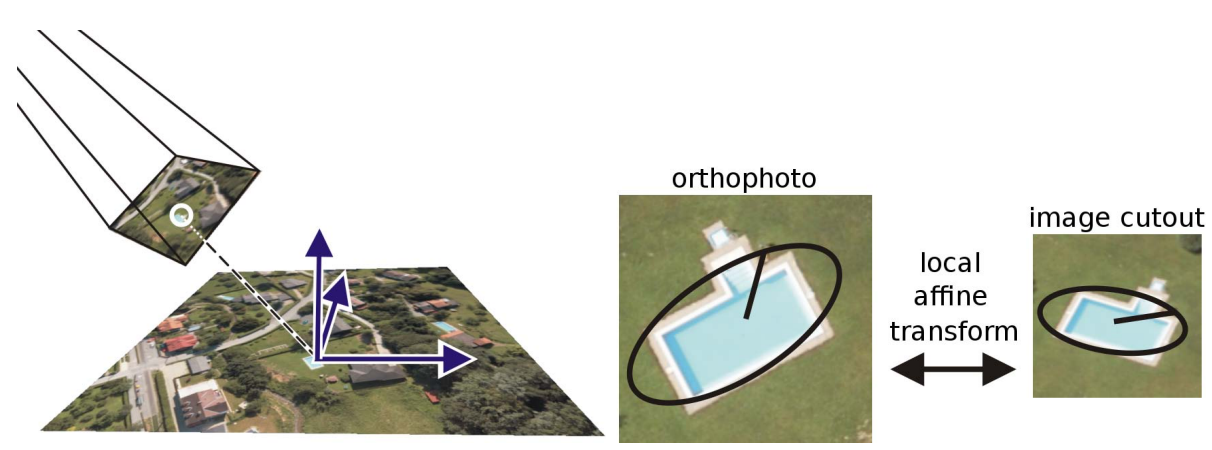

Fig. 1. Differential Spatial Resection exploiting Perspectivity. This figure shows an aerial camera observing a ground plane (left image). If the internal camera calibration is removed, the two images are related by a perspectivity. The projection of some point on the plane and the linear transform of the surrounding region provide 6 constraints for the 6 degrees of freedom for pose estimation. In the right part we see an MSER feature correspondence between an orthophoto and the unknown camera image providing a locally affine texture transform. The primitive for correspondence can be imagined as an infinitesimally small ellipse with orientation.

robot localization [29, initialization or recovery in camera tracking 3 [32] or determining the pose of a detected object 30. In these applications, often SIFT 18 or MSER 21] features are used nowadays, which cover some image region ideally corresponding to a surface in the scene. In 3] even the normal of such local surface regions is estimated and also 29] performs stereo from three cameras on a robot. However, in all of the above cited approaches, the correspondences are geometrically handled as points when it comes to initialization or direct pose estimation, although they carry much more information. Therefore, by now at least three of such robust feature correspondences were required to directly estimate a camera or object pose. In contrast, in this contribution we demonstrate how one affine image-model correspondence is already sufficient to estimate the pose.

The exploited primitive can also be seen as the limiting case where the three 3D points of Grunert's solution come infinitesimally close, allowing for what we call differential spatial resection. The concept of such correspondences has lately been proposed in [15] for estimation of the infinite homography and is displayed in fig 1. The question we answer is: Given a local affine transform between a region in some view and an orthophoto, how can we compute a homography with this transform as its local linearization and what camera pose belongs to it, given that the homography maps from a known world plane to the camera plane ? Furthermore, we show in section 4.1 the relation to pose estimation from conics, which is essentially a squared formulation of our approach although providing one degree of freedom less.

The proposed algorithm belongs to the set of minimal solvers, which exploit $n$ DOF (degrees of freedom) in some observation to estimate a model with also $n$ DOF. Such solutions are not targeted to produce ultimate optimal estimates 
but initial start values from as little data as possible, suitable for further processing. For instance, when dealing with small or hand-clicked data sets or when RANSAC-like estimators [6] are used, it is often desirable to obtain a minimal solution, which requires as little of the data as possible. In RANSAC, the probability of picking an all-inlier-set from correspondences with many mismatches depends exponentially on the number of samples required to construct a solution hypothesis. Using our novel approach, it is now possible to obtain an estimate of a camera's pose from as little as one e.g. MSER 21] or comparable feature (cf. to 24] for a discussion) or e.g. one suitable photogrammetric ground control point (cf. to 22, p.1111) in an image, given the local plane in 3D space where it is located and its texture.

For instance, when a feature descriptor is recognized in an unknown image, the 6 DOF camera or object pose can be obtained by the methods given here. To improve the pose estimation result, gradient based optimization techniques 1916] can be applied between the current view and a reference texture. The reference texture can either be an orthophoto (cf. to [22], p.758) or any other view with sufficient resolution for which the warp to an orthophoto is known. When several such feature correspondences and the camera poses are optimized at once, this is similar to the approach of Jin et al.11. However, their approach is formulated in a nonlinear fashion only and requires an initialization, comparable to the requirements for bundle adjustment. Since we exploit the perspectivity concept, a plane-to-plane mapping in euclidian space, in section 3 we also present the related work in homography estimation 331310 and projective reconstruction 27], which did not inspect the differential constraints on the perspectivity, because often the calibrated camera case is not considered in projective approaches. The exploitation of the Jacobian of the texture warp has been proposed though for the estimation of a conjugate rotation in [15].

Notation. To improve the readability of the equations we use the following notation: Boldface italic serif letters $\boldsymbol{x}$ denote Euclidean vectors while boldface upright serif letters $\mathbf{x}$ denote homogeneous vectors. For matrices we do not use serifs, so that Euclidean matrices are denoted as $A$ and homogeneous matrices are denoted as $\mathrm{A}$, while functions $\mathrm{H}[\boldsymbol{x}]$ appear in typewriter font.

\section{Perspectivity}

The contribution is based on estimating a transformation between two theoretical planes: The first plane is tangent to a textured surface in $3 \mathrm{D}$ and the second plane is orthogonal to the optical axis of a camera. The estimation of the pose is then formulated as the problem of obtaining a perspectivity between these two planes (see figure 1). A 2D perspectivity is a special kind of homography (cf. also to [9], pp. 34), which has only 6 degrees of freedom and which is particularly important for mappings between planes in Euclidian space. We assume a locally planar geometry at the origin of 3D space facing into z-direction and attach $x, y$-coordinates onto it, which coincide with the $x, y$ coordinates in $3 \mathrm{D}$ space. If we now move a perspective pinhole camera to position $C$ with orientation $R$ 
(which has rows $\boldsymbol{r}_{i}^{\top}$ ) and with internal camera calibration $\mathrm{K}$, a point $\mathbf{p}_{s}$ in space is mapped to an image point $\mathbf{p}_{i}$ by the camera as follows(cf. to [9], p. 157 for details):

$$
\mathbf{p}_{i}=\mathrm{K}\left(R^{\top} \mid-R^{\top} \boldsymbol{C}\right) \mathbf{p}_{s}
$$

We assume the internal parameters of our camera to be known and without loss of generality set $\mathrm{K}$ to the identity in the following. The method is not restricted to straight-line preserving ideal cameras, but can also be applied with real lenses with distortion, fish-eye lenses or even omni-directional cameras, as long as they have a single center of projection and the equivalent function of the matrix $\mathrm{K}$, which maps rays in the camera coordinate system to positions in the image, is differentiable and invertible.

Now we have a look at the points on our $z=0$ plane to derive the perspectivity:

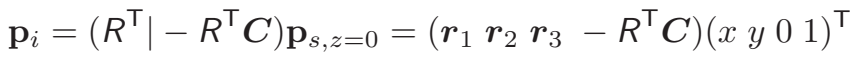

$$
\begin{aligned}
& =\left(\begin{array}{lll}
\boldsymbol{r}_{1} \boldsymbol{r}_{2}-R^{\top} \boldsymbol{C}
\end{array}\right)\left(\begin{array}{lll}
x & y & 1
\end{array}\right)^{\top} \simeq\left(\tilde{\boldsymbol{r}_{1}} \tilde{\boldsymbol{r}_{2}} \boldsymbol{t}\right)\left(\begin{array}{lll}
x & y & 1
\end{array}\right)^{\top}=\mathrm{H} \mathbf{p}_{p}
\end{aligned}
$$

$\tilde{\boldsymbol{r}}_{i}$ are scaled versions of $\boldsymbol{r}_{i}$ such that $\boldsymbol{t}_{z}=1$ and $\simeq$ means equality up to scale. Obviously, the homography $\mathrm{H}$ maps points $\mathbf{p}_{p}$ of the plane coordinate system to points $\mathbf{p}_{i}$ in the image coordinate system. $\mathrm{H}$ is a perspectivity and depends only on 6 parameters, the pose of the camera. Since $\mathrm{H}$ is an object of projective space, it can be scaled without changing the actual transformation. While the perspectivity $\mathrm{H}$ acts linearly in projective space $\mathbb{P}^{2}$, in Euclidian $2 \mathrm{D}$ space $\mathrm{H}$ is a nonlinear mapping from $\mathbb{R}^{2} \rightarrow \mathbb{R}^{2}$ because of the nonlinear homogenization:

$$
\mathrm{H}\left[\boldsymbol{p}_{p}\right]=\boldsymbol{p}_{i}=\frac{\left.\left(\mathrm{H} \mathbf{p}_{p}\right)\right|_{1 . .2}}{\left.\left(\mathrm{H} \mathbf{p}_{p}\right)\right|_{3}}
$$

In the next section we describe the differential correspondence and how it can be exploited to obtain constraints on $\mathrm{H}$.

\section{Differential Correspondence}

Progress in robust local features (cf. to 2423 for a thorough discussion) allows automatic matching of images in which appearance of local regions undergoes approximately affine changes of brightness and/or of shape, e.g. for automated panorama generation[1], scene reconstruction 30] or wide-baseline matching [18 21]. The idea is that interesting features are detected in each image and that the surrounding region of each feature is normalized with respect to the local image structure in this region, leading to about the same normalized regions for correspondences in different images, which can be exploited for matching. The concatenation of the normalizations provides affine correspondences between different views, i.e. not only a point-to-point relation but also a relative transformation of the local region (e.g. scale, shear or rotation). Although such correspondences carry more information than the traditional point 
correspondence used in estimation of multiple view geometry [9], this additional information is rarely used. Approaches not using point correspondences deal with conic correspondences [14]12, which typically lead to systems of quadratic equations or require lots of matrix factorizations. Schmid and Zisserman 28 investigated the behavior of local curvature under homography mapping. Chum et al. noted in 22] that an affine correspondence is somehow equivalent to three point correspondences: in addition to the center point two further points can be detected in the feature coordinate system (the local affine frame). This allowed the estimation of a fundamental matrix from 3 affine feature correspondences (from which 9 point correspondence were generated). A similar idea was also exploited recently in projective reconstruction, where the projection matrix was locally linearized 27] leading to additional constraints in non-linear optimization. The "local sampling" of the affine feature concept on the other hand was also adopted for other epipolar geometry problems, e.g. in [26. In contrast to the latter we do not sample but use a compact analytic expression for the whole correspondence: We observe that the concatenation of the normalization transformations provides a good approximation to the first order Taylor expansion of the perspectivity, i.e. that the resulting affine transform is the local linearization of the perspectivity, as it has been recently proposed for estimation of the infinite homography [15]:

$$
\begin{aligned}
\mathrm{H}[\boldsymbol{x}] & =\mathrm{H}\left[\boldsymbol{x}_{0}\right]+\left.\frac{\partial \mathrm{H}}{\partial \boldsymbol{x}}\right|_{\boldsymbol{x}_{0}}\left(\boldsymbol{x}-\boldsymbol{x}_{0}\right)+\ldots \\
A & \left.\approx \frac{\partial \mathrm{H}}{\partial \boldsymbol{x}}\right|_{\boldsymbol{x}_{0}} \quad A \in \mathbb{R}^{2 \times 2}
\end{aligned}
$$

Here $\mathrm{H}: \mathbb{R}^{2} \rightarrow \mathbb{R}^{2}$ is the homography mapping between the image and the orthophoto in Euclidean coordinates and $A$ represents local shear, scale and rotation between the two corresponding features. This fact has been exploited in matching for quite some time but has not been used for pose estimation before.

The considerations so far apply to affine features (e.g. MSER 21]). However, if matches result from weaker features (e.g. DoG/SIFT[18]), the proposed method can also be applied. The main insight is that if a correct match has been established such that the local regions are approximately aligned the affine transform based upon the relative parameters is already nearly correct.

However, since we need an accurate estimate of the Jacobian of the image transformation, it is reasonable even for already affine features to apply a gradient-based optimization of $A$ using the Lucas-Kanade approach [19]16. When using affine trackers, e.g. such as [3, the optimized information is readily available. We will call the point correspondence plus the local linear warp a differential correspondence in the remainder.

\section{Pose Estimation from a Differential Correspondence}

Having obtained a differential correspondence between a camera image and the textured plane in the origin, the local warp equals the derivative of the 
perspectivity. This derivative $\partial \mathrm{H} / \partial \boldsymbol{p}_{p}$ tells us something about the relative scaling of coordinates between the plane in the origin and the image, e.g. if $\boldsymbol{C}$ is large and the camera is far away from the origin $\partial \mathrm{H} / \partial \boldsymbol{p}_{p}$ will be small, because a large step on the origin plane will result in a small step in the image far away. Actually, $\partial \mathrm{H} / \partial \boldsymbol{p}_{p}$ carries information about rotation, scale and shear through perspective effects. Since $\mathrm{H}$ can be scaled arbitrarily without changing $\mathrm{H}$, we set $\mathrm{H}_{3,3}=1$ without loss of generality 11 and compute the derivative at the origin:

$$
\left.\frac{\partial \mathrm{H}}{\partial \boldsymbol{p}_{p}}\right|_{0}=\left(\begin{array}{l}
\tilde{r}_{11}-\tilde{r}_{13} t_{1} \tilde{r}_{12}-\tilde{r}_{13} t_{1} \\
\tilde{r}_{21}-\tilde{r}_{23} t_{2} \tilde{r}_{22}-\tilde{r}_{23} t_{2}
\end{array}\right)=\left(\begin{array}{ll}
a_{11} & a_{12} \\
a_{21} & a_{22}
\end{array}\right)
$$

Also, we compute where the origin is projected in our image:

$$
\mathbf{p}_{\text {origin }}=\mathrm{H}\left(\begin{array}{lll}
0 & 0 & 1
\end{array}\right)^{\top}=-R^{\top} \boldsymbol{C} \simeq \boldsymbol{t}
$$

Given a differential correspondence, the derivative as well as the projection of the origin are given by the relative parameters of the detected features. This can determine all degrees of freedom of the camera pose, however the overparameterization of the rotation must be resolved: Since $\tilde{R}$ is a scaled rotation matrix, $\tilde{\boldsymbol{r}}_{1}$ and $\tilde{\boldsymbol{r}}_{2}$ must be of same length and orthogonal:

$$
\tilde{r}_{11}^{2}+\tilde{r}_{12}^{2}+\tilde{r}_{13}^{2}=\tilde{r}_{21}^{2}+\tilde{r}_{22}^{2}+\tilde{r}_{23}^{2} \wedge \tilde{\boldsymbol{r}}_{1}^{\top} \tilde{\boldsymbol{r}}_{2}=0
$$

We can now compute $\mathrm{H}$ by first substituting $\boldsymbol{t}$ into eq. (7), then solving for $\tilde{r}_{11}, \tilde{r}_{21}, \tilde{r}_{12}$ and $\tilde{r}_{22}$ and substituting into eq.(9), leaving us with two quadratic equations in the two unknowns $\tilde{r}_{13}$ and $\tilde{r}_{23}$ :

$$
\begin{gathered}
\left(\tilde{r}_{13} t_{1}+a_{11}\right)^{2}+\left(\tilde{r}_{13} t_{1}+a_{12}\right)^{2}+\tilde{r}_{13}^{2}=\left(\tilde{r}_{23} t_{2}+a_{21}\right)^{2}+\left(\tilde{r}_{23} t_{2}+a_{22}\right)^{2}+\tilde{r}_{23}^{2} \\
\left(\tilde{r}_{13} t_{1}+a_{11}\right)\left(\tilde{r}_{23} t_{2}+a_{21}\right)+\left(\tilde{r}_{13} t_{1}+a_{12}\right)\left(\tilde{r}_{23} t_{2}+a_{22}\right)+\tilde{r}_{13} \tilde{r}_{23}=0
\end{gathered}
$$

The first equation is about the length and the second about the orthogonality of the $\tilde{r}$-vectors as typical for constraints on rotation matrices. We find it instructive to interpret them as the intersection problem of two planar conics, the length conic $\mathrm{C}_{l}$ and the orthogonality conic $\mathrm{C}_{o}$ :

$$
\begin{gathered}
\left(\tilde{r}_{13} \tilde{r}_{23} 1\right) C_{l}\left(\tilde{r}_{13} \tilde{r}_{23} 1\right)^{\top}=0 \\
\left(\tilde{r}_{13} \tilde{r}_{23} 1\right) C_{o}\left(\tilde{r}_{13} \tilde{r}_{23} 1\right)^{\top}=0 \\
C_{l}=\left(\begin{array}{ccc}
2 t_{1}^{2}+1 & 0 & t_{1}\left(a_{11}+a_{12}\right) \\
0 & -2 t_{2}^{2}-1 & -t_{2}\left(a_{21}+a_{22}\right) \\
t_{1}\left(a_{11}+a_{12}\right) & -t_{2}\left(a_{21}+a_{22}\right) & a_{11}^{2}+a_{12}^{2}-a_{21}^{2}-a_{22}^{2}
\end{array}\right) \\
C_{o}=\left(\begin{array}{ccc}
0 & t_{1} t_{2}+\frac{1}{2} & \left(a_{21}+a_{22}\right) t_{1} \\
t_{1} t_{2}+\frac{1}{2} & 0 & \left(a_{11}+a_{12}\right) t_{2} \\
\left(a_{21}+a_{22}\right) t_{1} & \left(a_{11}+a_{12}\right) t_{2} & a_{11} a_{21}+a_{12} a_{22}
\end{array}\right)
\end{gathered}
$$

${ }^{1}$ This is not a restriction because the only unrepresented value $\mathrm{H}_{3,3}=0$ maps the origin to the line at infinity and therefore such a feature would not be visible. 
Solving for the Pose Parameters. Two conics cannot have more than four intersection points, therefore, we can obtain at most four solutions for our camera pose. To solve the intersection of the two conics we use the elegant method of Finsterwalder and Scheufele [5], which proved also to be the numerically most stable method of the six different 3-point algorithms for spatial resection [8]: Since a common solution of equations (12) and (13) must also fulfill any linear combination of both, we construct a linear combination of both conics, which does not have full rank (zero determinant), but which still holds all solutions. This creates a third order polynomial, which has at least one real root and which can be solved easily:

$$
\operatorname{det}\left(\lambda \mathrm{C}_{o}+(1-\lambda) \mathrm{C}_{l}\right)=0
$$

The resulting degenerate conic will in general consist of two lines. The intersection of these lines with the original conics is only a quadratic equation and determines the solutions. The resulting $R$ and $\boldsymbol{C}$ have to be selected and normalized in such a way that we obtain an orthonormal rotation matrix (determinant +1 ) and the camera looks towards the plane. We have now obtained up to four hypotheses for the pose of the camera in the object coordinate system (relative to the feature). If there is a world coordinate system, in which the plane is not at the origin, the rigid world transformation has to be appended to the computed pose of the camera. Computing the relative pose in the object coordinate system in general also improves conditioning since the absolute numbers of the object's pose in the world become irrelevant.

Optimization and Tracking. Once initial parameters are obtained it is straightforward to use a 6-parametric gradient-based minimization technique 19 16] to further optimize the camera pose. Note that if we are using a pinhole camera and the feature in 3D is locally planar, instead of optimizing an approximate affine transform we might as well use a 6-parametric homography. Thus measurements may be incorporated from a larger region without making a mistake or an approximation. Even better, since it is possible to use global camera pose parameters, it is easy to optimize even multiple rigidly coupled features (e.g. in a rigid scene). Or, if robustness against outliers is a concern, each of the features provides an individual pose estimate and robust estimation techniques such as RANSAC 6] can be used to obtain a fused solution. If video data is available, the parameters can directly be used for tracking the regions, objects or camera pose over time similar to what is proposed in [11. However, in this contribution we focus on the geometric aspects of the minimal solution, i.e. where we see a single feature in a single image, without prior knowledge.

\subsection{Relation to Conic Correspondence}

In this section the differential feature concept is shown to be a simplified version of correspondences of conics, providing more constraints in a linear (instead of quadratic) fashion: In [20] Ma derived a way to determine the pose of a camera from two conics. He noted that a conic has only 5 DOF and thus a single conic is not sufficient to determine the 6 DOF of the camera pose uniquely. A conic 
$C_{S}$ on the space plane of the previous section maps to a conic $C_{I}$ in the image with the equation

$$
\mathrm{C}_{\mathrm{I}}=\mathrm{H}^{\top} \mathrm{C}_{\mathrm{S}} \mathrm{H} \text {, }
$$

where $\mathrm{H}$ is the perspectivity of the previous sections. First, we show how the two primitives used in our differential correspondence can be related to conic representations: For each affine feature, e.g. MSER, there exists a local image coordinate system, the local affine frame 2, such that coordinates can be specified relative to the size, shear, position and orientation of a feature. Imagine that $\mathrm{L}$ takes (projective) points from local feature coordinates to image coordinates:

$$
\mathrm{x}_{\mathrm{I}}=\mathrm{L} \mathrm{x}_{\mathrm{LAF}}
$$

If the same feature is seen in two images, points with identical feature (LAF) coordinates will have the same grey value. The local affine frames of the features in the different images are then called $L_{1}$ and $L_{2}$ and their concatenation is the first order Taylor approximation $\mathrm{H}_{\text {Taylor }}$ of the texture warp (e.g. a homography) between the two images at the feature positions:

$$
\mathrm{H}_{\text {Taylor }}=\mathrm{L}_{1} \mathrm{~L}_{2}^{-1}
$$

If we now just think of a single image and imagine a small ellipse through the points $(0 ; \lambda)^{\top},(\lambda ; 0)^{\top},(0 ;-\lambda)^{\top}$ and $(-\lambda ; 0)^{\top}$ of the local feature coordinate system, this ellipse can be represented by a conic equation in homogeneous coordinates such that points at the ellipse contour fulfill the quadratic constraint:

$$
0=\mathbf{x}_{\mathrm{LAF}}^{\top}\left(\begin{array}{ccc}
1 & & \\
& 1 & \\
& & -\lambda^{2}
\end{array}\right) \mathbf{x}_{\mathrm{LAF}}
$$

The LAF described as a conic matrix in image coordinates therefore is

$$
C_{\lambda}=L^{\top}\left(\begin{array}{lll}
1 & & \\
& 1 & \\
& & -\lambda^{2}
\end{array}\right) L=L^{\top} R^{\top}\left(\begin{array}{lll}
1 & & \\
& 1 & \\
& & -\lambda^{2}
\end{array}\right) R L
$$

where $\mathrm{R}$ is an arbitrary (homogeneous $2 \mathrm{D}$ ) rotation matrix, which cancels out. Therefore the first thing to observe is that $2 \mathrm{D}$ orientation of the feature is lost in conic representation. A conic has only five degrees of freedom and a conic correspondence therefore imposes at most five constraints on any $\mathrm{H}$. Furthermore, these constraints are quadratic in the entries of $\mathrm{H}$ as can be seen from eq. (17). This equation is also essentially a squared version of equation (19). On the other hand, the differential correspondence is only valid locally and introduces inacurracies for larger regions, but it is available when sufficient texture is in the image, while a conic may have any size. However, conics traditionally exploit a special geometric shape (typically an ellipse contour) and ideal perspective cameras and ideal planes, because conic curve estimation in distorted cameras is more involved. In contrast, the differential feature concept can also directly be applied in fish-eye or omnidirectional cameras. 


\section{Evaluation}

In this section the differential correspondence-based pose estimation is evaluated first using synthetic sensitivity experiments. Next, rendered images with known ground truth information are used to evaluate the real-world applicability, where everything has to be computed from image data. In the final experiments, object pose estimation from one feature is shown qualitatively using non-ideal cameras.

Sensitivity to Noise and Internal Calibration Errors. Our evaluation starts with an analysis of the sensitivity to different disturbances. Since the algorithm provides a minimal solution, which translates a 6 DOF differential correspondence into a 6 DOF pose, the pose will adapt to noise in the correspondence. In figure (5) it is shown that for localization accuracies better than 1 pixel in a camera with focal length 500 pixel the camera orientation is on average better than 1 degree and also the direction of the camera center is better than 1 degree. The orientation error is computed from the axis-angle representation of the rotation which transforms the ground truth orientation into the estimated orientation and therefore incorporates all directions. The center error is the angle between the ground truth camera center and the estimated camera center as seen from the 3D feature's position.

To obtain a reasonable noise magnitude for the differential correspondence parameters, we assume that the center of a patch can be localized with a Gaussian uncertainty of zero mean and variance $\sigma_{p}^{2}$ and that the corners of a square patch of size $(2 w+1) \times(2 w+1)$ pixels can be localized with about the same uncertainty, which can then be propagated to uncertainty for the affine parameters. When creating noisy $6 \mathrm{D}$ affine features, we therefore sample the noise from a Gaussian distribution with diagonal covariance depending on one parameter $\sigma_{p}$, which is printed on the x-axis of figure 5 . It is remarkable that the errors in orientation and position are highly correlated. This can be explained from the fact that a slightly different differential correspondence results in a slightly different camera orientation. However, since the feature must be projected to about the same position, the camera center has to adapt accordingly. As figure 5 shows, the pose estimation is stable even when the camera is not calibrated correctly, although it can be seen that the resulting pose is disturbed as inherent in minimal solutions. In particular it is clear that an error in principal point results in an error in the pose when the reference feature in 3D is correct. Keep in mind that at focal length 500 a principal point error of ten pixel means that the optical axis is more than $1^{\circ}$ mis-calibrated.

\section{Solid Angle, Approximation by 3 Points and Comparison with Spa-} tial Resection/POSIT. Using the proposed approach, the affine warp must be measured between the orthophoto and the image under inspection and this requires a region upon which this is done. If the alignment is done using an affine warp, the region should be chosen as small as possible, particularly when the feature is seen from an oblique angle, because in the affine warp model it is assumed that the warp (the Jacobian of the homography) does not change between the 

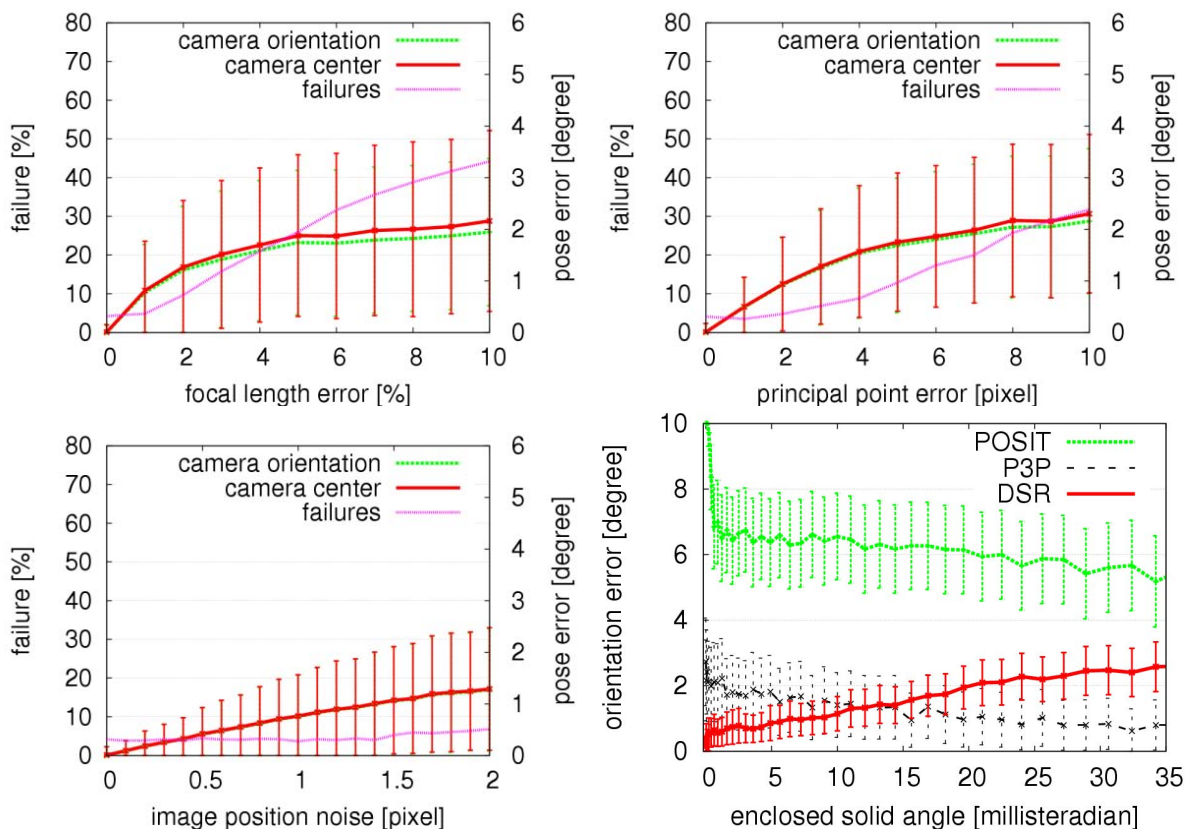

Fig. 2. Sensitivity with Respect to Noise, Calibration and Feature Area. In these experiments, 105.000 random camera poses in front of the $z=0$ plane have been synthesized (providing the ground truth differential correspondences). In the two top graphs, focal length $(500)$ and principal point $(100 ; 100)$ have been disturbed up to $10 \%$ and the resulting error in the best pose is displayed as well as the number of cases, where no solution was possible (or the best pose was more than $5^{\circ}$ off). In the lower left graph, Gaussian noise has been added to the 6 parameters of the differential correspondence, where we assume that the position accuracy $\sigma_{p}$ of the center of the patch is the same as for the corners of a patch of half window size $w$ and therefore disturb the 4 affine parameters with $\sigma_{p} /(\sqrt{2} w)$, where we assume a $21 \times 21$ window. The error bars indicate the size of the standard deviation. In the bottom right figure, we compare the 3-point solution proposed in the Manual of Photogrammetry [22, pp.786] (P3P), the planar POSIT algorithm 25] based on the 4 patch corners (which already includes the parallel projection approximation by [17] in the POS step) and our novel solution applied to the case that we use 3 or more close points: The differential correspondence is approximated using the four patch corners only, while we vary the size (given as the solid angle) of the patch and fix $\sigma_{p}$ for the corners at 0.5. The error bars show $1 / 3$ standard deviation. As expected, it can be seen that for large solid angle spatial resection performs best while for decreasing solid angles the novel solution gets better and better, outperforming the other approaches for very narrow constellations.

corners of the local patch. On the other hand, when the 3 individual 3D points of Grunert's solution approach each other, the standard spatial resection can become unstable, because it is based on the difference of the distances to the 3 points. To overcome this issue, Kyle 17] proposed an approximate initial guess 

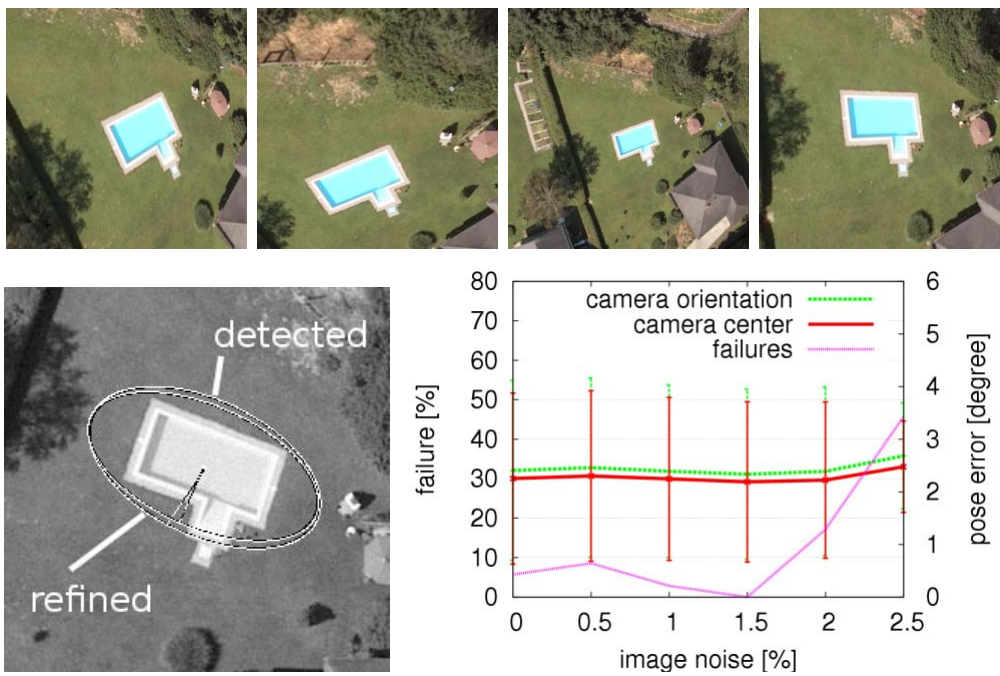

Fig. 3. Camera Pose From Noisy Images. A ground plane has been textured with an aerial image serving as an orthophoto and a series of 40 views have been rendered with different levels of noise (upper row: sample views with low noise). A reference MSER feature with orientation has been chosen in the orthophoto. This feature is then detected in the other views and refined using a simple 6-parametric affine warp (see ellipses in bottom left image) according to [16] based upon a half window size of 10 pixels. From such differential correspondences, the camera pose is estimated and compared against the known ground truth value as explained earlier. Whenever the error was above $20^{\circ}$ or the algorithm did not come up with a solution a failure was recorded. The bottom right graph shows the average pose errors in dependence of the added image noise. When adding much more image noise, the MSER detector is no longer able to find the feature. This experiment is particularly interesting because it shows that the concept does still work when the ellipse is not infinitely small.

for narrow angle images, which is the same as the POS (Pose from Orthography and Scaling) in the POSIT 4 algorithm: Both require 4 non-coplanar points. For the POSIT algorithm however, there exists also a planar variant 25], which copes with planar 3D points.

Therefore we compare our novel algorithm (well-suited for small solid angles) to the spatial resection [78] implemented as proposed in the Manual of Photogrametry 22, pp. 786] and the planar POSIT 25] algorithm kindly provided on the author's homepage, which are both desiged for larger solid angles. We vary the size of a local square image patch from ten to several hundred pixels and use the corners as individual 2D-3D correspondences in the existing algorithms. For our new method the patch corner points are used to compute a virtual local affine transform which approximates the required Jacobian. An evaluation of the quality of the approximation can be seen in the bottom right of fig. 5. which shows that for small solid angles the novel solution outperforms spatial resection, while for large solid angles - as expected - the affine 

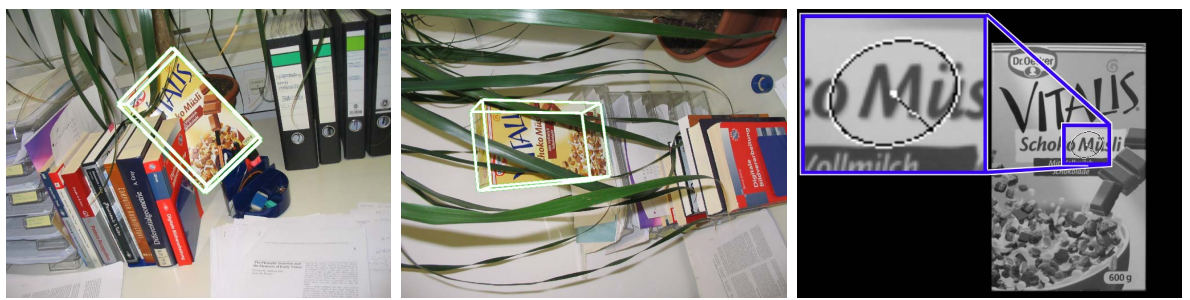

Fig. 4. Object Pose Estimation from a Single Feature. This figure shows that in a real camera with radial distortion object pose estimation is possible from a single feature. The orthophoto of the object is displayed in the right image with the local feature region enlarged. The two left images show cluttered views with the object partially occluded. The "M" has been detected using MSER and refined, the resulting object poses from this single differential correspondence are then displayed by augmenting a contour model (white).

approximation is not suitable. It is however still better in average than the orthographic approximation in the planar POSIT algorithm. Particularly, when the solid angle approaches zero, the error in the novel solution tends to zero, while for the other algorithms no solution can be obtained or the best solution is worse than the robust error threshold of $10^{\circ}$.

Normal or Pose Error of the Local Plane. An error of the normal of the $3 \mathrm{D}$ reference plane, for which the orthophoto exists or an error of the pose of this plane cannot be detected within the algorithm. The pose is computed relative to this plane and an error of the plane in global coordinates will consequently result in a relative error of the camera pose in global coordinates.

Warp Measured From Real Texture. In the next experiment, we rendered views from a ground plane and apply automatic matching and pose estimation based upon a single prominent image feature. Since we do have the ground truth data the estimated pose can be analyzed in this case. The experiments are explained in fig 3 and show that even in presence of image noise using automatic matching and refinement approaches the pose can be estimated quite reliably, given the minimal local texture data which is used.

Images with Radial Distortion. In the final experiment we took photographs of an office scene, where we detect a cereal box, which is partially occluded. As in the previous experiment, an MSER feature is obtained from an orthophoto of the cereal box. Next this feature is automatically found in the test image and refined using gradient-based (affine) optimization. Again, from this differential correspondence the object pose is estimated, which might be interesting in applications where only small portions of an object are visible. The augmentation in fig:4 shows quite good results. 


\section{Conclusion}

A method for estimating a camera pose based upon a single local image feature has been proposed which exploits the often readily available local affine warp between two images. This differential correspondence provides more constraints than a point or a conic and can be used easily in calibrated cameras even if they deviate from the linear projection model. The algorithm proved to be stable under several kinds of disturbance and can also be applied when the 3 individual $3 \mathrm{D}$ points of a general spatial resection problem come very close because the novel formulation avoids directly computing the 3 distances, which can lead to numerical difficulties in practise. Another benefit of the novel minimal solution is that it allows now for computing the pose from a single image-model match of common robust features which could reduce RANSAC complexity compared to the previously required set of 3 correspondences.

\section{References}

1. Brown, M., Lowe, D.G.: Automatic panoramic image stitching using invariant features. International Journal of Computer Vision 74(1), 59-73 (2007)

2. Chum, O., Matas, J., Obdrzalek, S.: Epipolar geometry from three correspondences. In: Computer Vision Winter Workshop, Prague, pp. 83-88 (2003)

3. Davison, A.J., Reid, I.D., Molton, N.D., Stasse, O.: Monoslam: Real-time single camera slam. IEEE Transactions on Pattern Analysis and Machine Intelligence 29(6), 1052-1067 (2007)

4. DeMenthon, D., Davis, L.S.: Model-based object pose in 25 lines of code. International Journal of Computer Vision 15, 123-141 (1995)

5. Finsterwalder, S., Scheufele, W.: Das Rückwärtseinschneiden im Raum. In: Bayerische, K., der Wissenschaften, A. (eds.) Sitzungsberichte der mathematischphysikalischen Klasse, vol. 23/4, pp. 591-614 (1903)

6. Fischler, M., Bolles, R.: RANdom SAmpling Consensus: a paradigm for model fitting with application to image analysis and automated cartography. Communications of the ACM 24(6), 381-395 (1981)

7. Grunert, J.A.: Das Pothenot'sche Problem, in erweiterter Gestalt; nebst Bemerkungen über seine Anwendung in der Geodäsie. In: Archiv der Mathematik und Physik, vol. 1, pp. 238-248, Greifswald. Verlag C.A. Koch (1841)

8. Haralick, B., Lee, C., Ottenberg, K., Nölle, M.: Review and analysis of solutions of the three point perspective pose estimation problem. International Journal of Computer Vision 13(3), 331-356 (1994)

9. Hartley, R., Zisserman, A.: Multiple View Geometry in Computer Vision, 2nd edn. Cambridge University Press, Cambridge (2004)

10. Irani, M., Rousso, B., Peleg, S.: Recovery of ego-motion using region alignment. Transact. on Pattern Analysis and Machine Intelligence 19(3), 268-272 (1997)

11. Jin, H., Favaro, P., Soatto, S.: A semi-direct approach to structure from motion. The Visual Computer 19(6), 377-394 (2003)

12. Kahl, F., Heyden, A.: Using conic correspondence in two images to estimate the epipolar geometry. In: Proceedings of ICCV, pp. 761-766 (1998)

13. Kähler, O., Denzler, J.: Rigid motion constraints for tracking planar objects. In: Hamprecht, F.A., Schnörr, C., Jähne, B. (eds.) DAGM 2007. LNCS, vol. 4713, pp. 102-111. Springer, Heidelberg (2007) 
14. Kannala, J., Salo, M., Heikkila, J.: Algorithms for computing a planar homography from conics in correspondence. In: Proceedings of BMVC 2006 (2006)

15. Koeser, K., Beder, C., Koch, R.: Conjugate rotation: Parameterization and estimation from an affine feature corespondence. In: Proceedings of IEEE Conference on Computer Vision and Pattern Recognition (CVPR) (2008)

16. Koeser, K., Koch, R.: Exploiting uncertainty propagation in gradient-based image registration. In: Proc. of BMVC 2008 (to appear, 2008)

17. Kyle, S.: Using parallel projection mathematics to orient an object relative to a single image. The Photogrammetric Record 19, 38-50 (2004)

18. Lowe, D.G.: Distinctive image features from scale-invariant keypoints. International Journal of Computer Vision 60(2), 91-110 (2004)

19. Lucas, B.D., Kanade, T.: An iterative image registration technique with an application to stereo vision. In: IJCAI 1981, pp. 674-679 (1981)

20. De Ma, S.: Conics-based stereo, motion estimation, and pose determination. International Journal of Computer Vision 10(1), 7-25 (1993)

21. Matas, J., Chum, O., Urban, M., Pajdla, T.: Robust wide baseline stereo from maximally stable extremal regions. In: Proceedings of BMVC 2002 (2002)

22. McGlone, J.C. (ed.): Manual of Photogrammetry, 5th edn. ASPRS (2004)

23. Mikolajczyk, K., Schmid, C.: A performance evaluation of local descriptors. Transact. on Pattern Analysis and Machine Intell. 27(10), 1615-1630 (2005)

24. Mikolajczyk, K., Tuytelaars, T., Schmid, C., Zisserman, A., Matas, J., Schaffalitzky, F., Kadir, T., van Gool, L.: A comparison of affine region detectors. International Journal of Computer Vision 65(1-2), 43-72 (2005)

25. Oberkampf, D., DeMenthon, D., Davis, L.S.: Iterative pose estimation using coplanar feature points. CVGIP 63(3) (1996)

26. Riggi, F., Toews, M., Arbel, T.: Fundamental matrix estimation via TIP - transfer of invariant parameters. In: Proceedings of the 18th International Conference on Pattern Recognition, Hong Kong, August 2006, pp. 21-24 (2006)

27. Rothganger, F., Lazebnik, S., Schmid, C., Ponce, J.: Segmenting, modeling, and matching video clips containing multiple moving objects. IEEE Transactions on Pattern Analysis and Machine Intelligence 29(3), 477-491 (2007)

28. Schmid, C., Zisserman, A.: The geometry and matching of lines and curves over multiple views. International Journal of Computer Vision 40(3), 199-234 (2000)

29. Se, S., Lowe, D.G., Little, J.: Vision-based global localization and mapping for mobile robots. IEEE Transactions on Robotics 21(3), 364-375 (2005)

30. Skrypnyk, I., Lowe, D.G.: Scene modelling, recognition and tracking with invariant image features. In: IEEE and ACM International Symposium on Mixed and Augmented Reality, pp. 110-119 (2004)

31. Thompson, E.H.: Space resection: Failure cases. The Photogrammetric Record 5(27), 201-207 (1966)

32. Williams, B., Klein, G., Reid, I.: Real-time slam relocalisation. In: Proceedings of ICCV, Rio de Janeiro, Brazil, pp. 1-8 (2007)

33. Zelnik-Manor, L., Irani, M.: Multiview constraints on homographies. IEEE Transactions on Pattern Analysis and Machine Intelligence 24(2), 214-223 (2002) 